\title{
Functional Connectivity within Brain Networks of Long Term and Short term Meditators
}

\author{
Ashwini S Savanth, P.A.Vijaya, Bindu M. Kutty
}

\begin{abstract}
Meditation refers to a state of mind of relaxation and concentration, where generally the mind and body is at rest. The process of meditation reflects the state of the brain which is distinct from sleep or typical wakeful states of consciousness. Meditative practices usually involve regulation of emotions and monitoring of attention. Over the past decade there has been a tremendous increase in an interest to study the neural mechanisms involved in meditative practices. It could also be beneficial to explore if the effect of meditation is altered by the number of years of meditation practice. Functional Magnetic Resonance Imaging (fMRI) is a very useful imaging technique which can be used to perform this analysis due to its inherent benefits, mainly it being a non-invasive technique. Functional activation and connectivity analysis can be performed on the fMRI data to find the active regions and the connectivity in the brain regions. Functional connectivity is defined as a simple temporal correlation between anatomically separate, active neural regions. Functional connectivity gives the statistical dependencies between regional time series. It is a statistical concept and is quantified using metrics like Correlation. In this study, a comparison is made between functional connectivity in the brain regions of long term meditation practitioners (LTP) and short-term meditation practitioners (STP) to see the differences and similarities in the connectivity patterns. From the analysis, it is evident that in fact there is a difference in connectivity between long term and short term practitioners and hence continuous practice of meditation can have long term effects.
\end{abstract}

Keywords: fMRI, functional connectivity, meditation, meditation experience.

\section{INTRODUCTION}

Meditation techniques help in transforming consciousness. Meditation helps in gaining higher insight by introspectively observing and giving attention to the thought processes. It has also become very promising as an alternative and complementary therapy for various diseases and ailments [1]. Meditation refers to a state of mind of relaxation and concentration, where generally the mind and body is at rest. The process of meditation reflects the state of the brain which is distinct from sleep or typical wakeful states of consciousness. Meditative practices usually involve regulation of emotions and monitoring of attention. Over the past decade there has been a tremendous increase in interest to study the neural basis of meditative practices. Indeed, it has become very evident with the various studies carried out, that

Revised Manuscript Received on December 12, 2019.

* Correspondence Author

Ashwini S Savanth*, Department of ECE, BNM Institute of Technology, VTU, Bangalore, India. Email: ashwini.savanth@gmail.com

Dr. P.A.Vijaya, Department of ECE, BNM Institute of Technology, VTU, Bangalore, India. Email: pavmkv@gmail.com

Dr. Bindu M. Kutty, Department of Neurophysiology, NIMHANS, Bangalore, India. Email: bindu.nimhans@gmail.com meditation brings about significant changes in cognitive and affective processing. Meditation is also found to alter the brain structure and function, which is evident to the fact that the human brain is like a river and not a rock. Meditation is found to be beneficial as it helps calm the mind and body. It has also been found that in many meditation practices, individuals feel calmer and find a dip in body metabolism. Another very important advantage of meditation is that it helps to improve one's attention. All these findings lead to a valuable question as to "What might be going on in the mind and thus the brain of a meditator to produce these behavioral effects?" This question has fascinated the psychologists, neuroscientists and the meditators alike. It seems to be inspiring to study the meditating brain to learn how it changes, how it works and to find better therapies for body-mind health [1]. Also, there is a need to explore if the effect of meditation is altered by the number of years of meditation practice. In this study, a comparison is made between long term meditation practitioners (LTP) and short-term meditation practitioners (STP) to see the differences and similarities in the connectivity patterns. In this experimental paradigm, the subjects perform a task called the Assessing Neurocognition via Gamified Experimental Logic (ANGEL) [2]. The ANGEL paradigm has an audio visual sensory motor design and allows assessment of 10 neurocognitive functions. It aids in the assessment of several levels of decision making in presence of multiple distractors.

\section{FMRI AS AN IMAGING MODALITY}

Scientists are imaging the brain and made pictures of the brain activity and recorded the brain waves of thousands of meditators practicing different forms of meditation. They are investigating to see if the benefits of meditation as experienced by the meditators like increased calmness, reduced stress, better attention, can be traced to the changes in the neural mechanisms. There are pioneers in the field of meditation research like Dr. Herbert Benson and Jon Kabat-Zinn, and scientists like Richard Davidson and Sara Lazar who have used modern research tools in order to study the effects of meditation on brain and health. Several brain imaging and signaling techniques like Positron Emission Tomography (PET), Magnetic Resonance Imaging (MRI), functional Magnetic Resonance Imaging (fMRI), Electroencephalogram (EEG), Magnetoencephalography (MEG) have been used in order to understand this effect of meditation on the brain. fMRI is a very useful imaging technique which can be used to perform this analysis due to its inherent benefits, mainly it being a non-invasive technique and hence the subject will not be exposed to any harmful radiation during the experimental process. 
It also has a good balance between spatial resolution and temporal resolution, giving it an added advantage. In an fMRI experiment the subject performs a task during which a series of brain images are acquired. By measuring the variations in the signal between individual images, inferences can be made on the task related activations. The Blood Oxygenation Level Dependent (BOLD) contrast is the most common measure of fMRI. BOLD fMRI measures the ratio of oxygenated to deoxygenated hemoglobin in the blood. BOLD fMRI does not directly measure the neuronal activity, but it measures the oxygen consumed by the active neurons i.e. the metabolic demand of neurons. The hemodynamic response function (HRF) represents changes in the fMRI signal triggered by neuronal activity. fMRI data analysis is a massive data problem. Each brain volume consists of approximately 100,000 voxel measurements. Each experiment consists of hundreds of brain volumes. Each experiment may be repeated for multiple subjects to facilitate population inference. The total amount of data that needs to be analyzed is staggering. Also, the signal of interest is relatively weak, and it exhibits a complicated temporal and spatial noise structure. These factors make statistical analysis of fMRI data challenging. In fMRI analysis, functional activation analysis helps to find the regions of the brain that are activated due to a task or at rest and functional connectivity analysis is used to find the connectivity (structural/ functional/ effective) between these regions. The experimental design of fMRI analysis usually follows the following steps: data acquisition, reconstruction, pre-processing, data analysis, localizing brain activity, connectivity and prediction.

The brain is always active irrespective of explicit input or output. Spontaneous BOLD activity occurs during task and at rest. Only 5\% of the brain's total energy is consumed in the neuronal metabolism due to task related changes. Neuroscientists are interested in studying the spontaneous BOLD signals of resting state networks which gives the correlation between brain regions that are structurally and/or functionally related. This will help in learning the intrinsic functional connectivity in the brain. Hence, resting state fMRI has found to be very useful in the study of brain connectivity. Some of the Resting State Networks (RSNs) are Dorsal Attention Network (DAN), Ventral Attention Network (VAN), Sensori Motor Network (SMN), Visual network (VIS), Fronto- Parietal Control Network (FPC), Language Network (LAN), Default Mode network (DMN). The RSNs show activity during rest and during tasks. The DMN shows a decrease in activity during cognitive tasks.

\section{LITERATURE SURVEY}

Very few studies have been carried out so far where a difference in connectivity in the brain regions are seen due to meditation experience. Baron Short et.al. reported that the time courses of long-term practitioners had much better consistency and sustained activation in the dorsal lateral prefrontal cortex (DLPFC) and anterior cingulate cortex (ACC) regions of the brain [3]. J.A. Brefczynski-Lewis et. al. reported that the prefrontal cortex of long term practitioners showed a lot more activations during meditation than that of short term practitioners [4]. Holzel et.al. reported that the anterior cingulate and medial prefrontal cortex (MPFC) of 15 experienced meditators showed more activations than those of control subjects with no experience of meditation [5]. Joon Hwan Jang et.al. have indicated that the long-term practice of meditation may be associated with functional changes in regions related to internalized attention even when meditation is not being practiced [6].

\section{MATERIALS AND METHODS}

\section{A. Participants}

fMRI data used in this study was acquired at NIMHANS, Centre for Consciousness studies. The data acquisition was performed by Ajay $\mathrm{K}$ Nair et.al. at NIMHANS, Bangalore [2]. Two groups of people were recruited for data acquisition, 12 Long Time Practitioners (LTP) who had at least 10 years of Rajayoga meditation practice and 10 Short Term Practitioners (STP) who had about one year of practice. The participants were matched for age, gender, education and monthly family income. They were from diverse socio-economic conditions with diverse education levels. All participants were healthy, right-handed and non smokers.

\section{B. Image acquisition and fMRI protocol}

fMRI data were acquired using 3T Siemens SKYRA MRI scanner. The scan parameters were $\mathrm{TR}=2000 \mathrm{~ms}$, $\mathrm{TE}=$ 30ms. fMRI protocol followed was rest, task, rest. The task was a modified version of the 'Who' task which is the second level of ANGEL (Assessing Neurocognition via Gamified Experimental Logic) paradigm [2]. The ANGEL is a neurocognitive paradigm which was developed for performing a parametric evaluation of multiple neurocognitive functions simultaneously. It is a gamified adaptation of the visual oddball paradigm. For the fMRI version of the task, the trial length was increased to suit a TR of $2000 \mathrm{~ms}$ and a few blocks contained passive trials (with no responses) to provide an additional baseline to compare with the rest of the trials. It is a mixed design experiment with sets of events coming in blocks with different regularity. There were 16 blocks, each block comprising of 25 stimulus trials and 3 baseline trials and hence a total of 448 trials. The task provided opportunities to study cognition across different event sets (rare versus frequent events, face perception vs shape perception, active response vs passive observation to task etc.)

Before the trial starts, a white + sign appears on the screen which has a gray background with black and white checkerboards on either side of the + sign. During the trials, one of the checker boards is replaced with one of the four visual stimuli in a pseudorandom manner for a duration of $240 \mathrm{~ms}$. The visual stimuli is black and white and is one of: Kanizsa triangle or a distorted version of it which is formed with components of Kanizsa triangle or Mooney face or a distorted version of it. One of four salient image is presented on one side of the + sign for $80 \%$ of the time, this is the Frequent image category while two other image types are presented on the other side for a total $20 \%$ of the time, forming the Rare category.

Out of the total 16 blocks, there are 8 odd blocks and 8 even blocks. During the 'Who' 
task, participants must give a button response when the salient stimuli is presented during all blocks. But, in this level, for half of the pseudo randomly allocated blocks, a brief polysyllabic tone is immediately generated by the system when the subject makes a button response. For the remaining blocks, the tone is generated after the stimulus is presented with a randomly introduced delay. For the latter blocks, if the subject does not make a response, the tone is generated after the timeout period.

\section{Image pre-processing}

Data was pre-processed with the CONN toolbox [7] (http://www.nitrc.org/projects/conn) which implements all the pre-processing steps in SPM12 software (http://www.fil.ion.ucl.ac.uk/spm/). Slice time correction and realignment were performed. Motion parameters were regressed out. The realigned images were then normalized to the MNI template. Also, segmentation of gray matter, white matter and CSF areas were performed for optionally using it during the removal of temporal confounding factors. The images were then smoothed with a Gaussian kernel of $8 \mathrm{~mm}^{3}$ FWHM. Additional preprocessing steps like bandpass filtering, including additional covariates like white matter and CSF time series, respiratory and cardiac signals, estimated subject motion parameters and artifacts were included here which help reduce the impact of motion and physiological noise factors. This will increase the robustness of fcMRI analysis and reduces false positives. In addition, the Artifact Detection Tools (ART: http://www.nitro.org/projects/ artifact_detect) were used to measure motion artefacts in all individuals in both groups; any motion artefacts were controlled by realignment parameters detected with ART.

\section{Functional connectivity analysis}

A region of interest (ROI) based functional connectivity analysis was performed using the CONN toolbox. In the first level analysis, functional connectivity measures for each subject and for each condition was evaluated. Seed and target ROIs that were selected include 32 areas of the cortex from 8 networks which are Default mode (DMN), Sensorimotor, Visual, Salience, Dorsal Attention (DAN), Frontoparietal, Language, Cerebellar. The BOLD time series of any given ROI was estimated by averaging the BOLD time series across all the voxels in the ROI. A weighted General Linear Model for weighted regression/correlation measures of the condition-specific association between the seed/source BOLD timeseries and each voxel or target ROI BOLD timeseries were performed. The Fischer Z-transformation was calculated from the correlation coefficients between each ROI to ROI BOLD time series pair resulting in a $32 \times 32$ connectivity matrix. In the second level analysis, both between-condition and between-source contrasts represent within-subject effects. Second-level analyses will correspond to multivariate/repeated-measures analyses of the selected effects. Second-level model will be a general linear model that includes as regressors the selected terms in the "Subject effects" list. The outcome variable will be the within-subjects linear combination(s) of effects specified by the "between-conditions" and "between-sources" contrasts, applied to the first-level connectivity-measure matrix. The graphical display and second level results are thresholded using FDR correction p-value of 0.05 and is set to display two sided results. The general linear model is defined as given in equation 1

$$
\text { data fit: } \boldsymbol{Y}=\boldsymbol{X}^{*} \boldsymbol{B}
$$

$$
\text { hyp test: } \boldsymbol{C}^{*} \boldsymbol{B} * \boldsymbol{M} \boldsymbol{\prime}=\mathbf{0}
$$

$\mathrm{C}->$ Columns of $\mathrm{X}$ (effects) (between subjects contrast)

$\mathrm{M} \rightarrow$ Columns of $\mathrm{Y}$ (conditions) (between conditions contrast)

\section{RESULTS AND DISCUSSION}

\section{A. Main effects of LTP and STP for one condition}

A matrix of correlation coefficients between the 32 regions of interest thresholded at $\mathrm{pFDR}<0.05$ was formed for one condition (shapepresent) for LTP and STP separately. The connectivity matrix is shown in figure 1 and 2 . The cells are colour coded for better understanding, yellow is for connectivity between same ROIs, red indicates positive value and blue indicates negative values of correlation. Empty cells indicate no significant connectivity. A global functional connectivity (GFC) measure which is defined as the number of elements in a row/column with absolute Z-score greater than a threshold was found for LTP and STP. A threshold of $\mathrm{z}=0.424$ was used and the GFC measure was found for all the regions. It was found that the connectivity in all the regions were almost similar except for two regions the Anterior Cingulate Cortex (ACC) which is part of the salience network and Intra Parietal Sulcus Right (IPS(R )) which is part of the Dorsal Attention Network. IPS(R ) had a GFC measure of 5 in LTP as compared to a value of 2 in STP. The DAN is active during the execution of a task and is involved in working memory and sustained attention. ACC on the other hand had a GFC value of 0 in LTP against a value of 4 in STP. ACC is associated with self regulation, emotional regulation, self control and attention. On one hand there is a good connectivity in IPS and no connectivity in ACC for LTP. So, it is not very easy to conclude that LTP has better attention capability than STP but, there seems to be a difference in connectivity pattern between LTP and STP. The results shown here are obtained only for one condition but the other conditions also need to be considered and then a valid conclusion could be drawn.

\section{B. Comparison of main effect of LTP and STP for different contrasts in conditions}

Table 1 shows a comparison of main effect of LTP and STP for different contrasts in conditions. No significant connectivity is found in STP for two contrasts i.e. rare > frequent and face absent $>$ face present. Whereas no significant connectivity is found in LTP for the contrast shape present > shape absent. From this comparison it is evident that there is a difference in the connectivity between regions for LTP and STP. These features can be used to train a classifier in order to classify long term and short term meditation practitioners.

\section{Visualization of the connectivity pattern}

CONN toolbox can be used to visualize the connectivity pattern. Figure 3 and 4 shows the connectivity pattern 
obtained between the 32 ROIs for LTP and STP respectively.

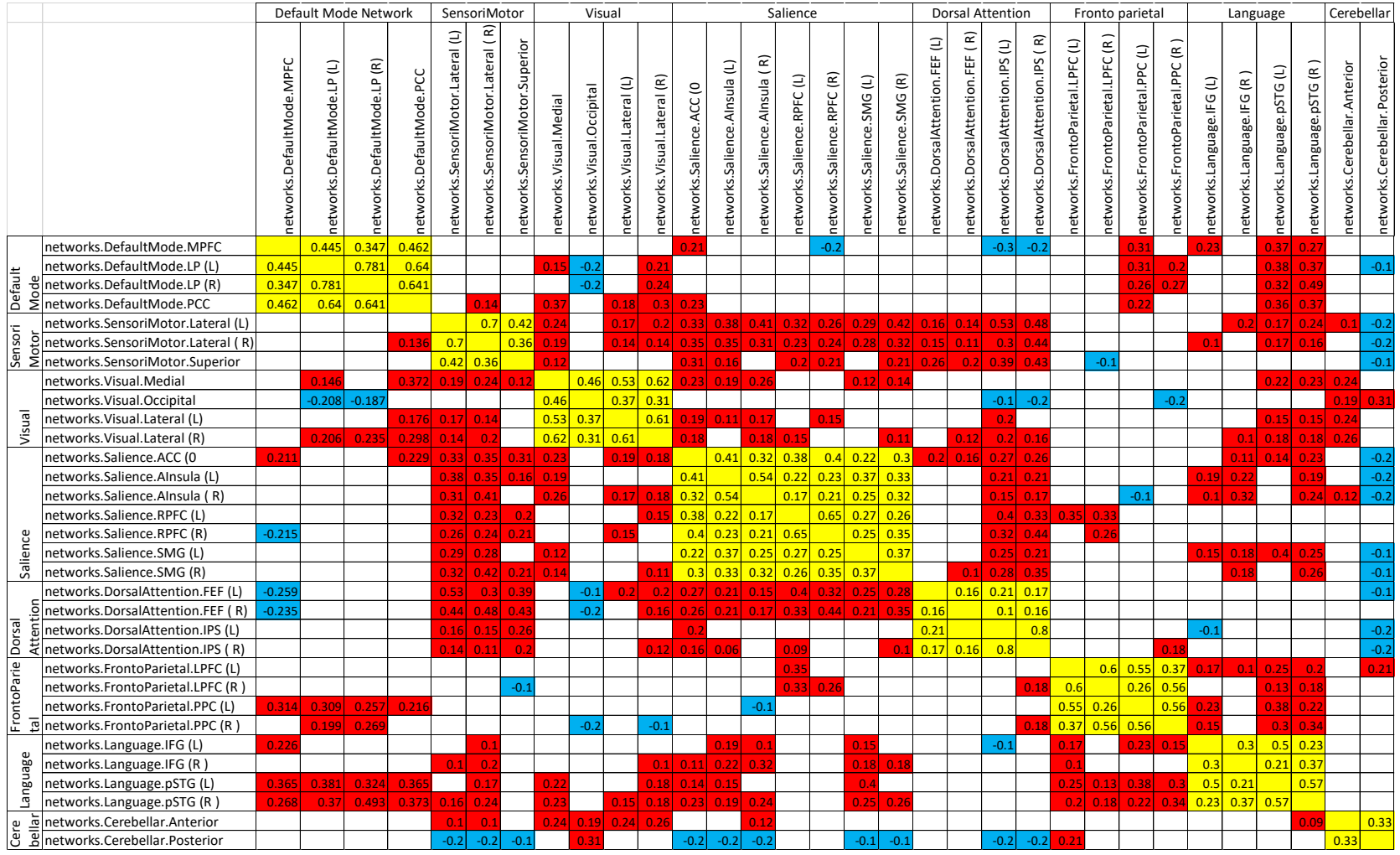

Fig. 1: Connectivity matrix for Main effects of LTP for condition shapepresent

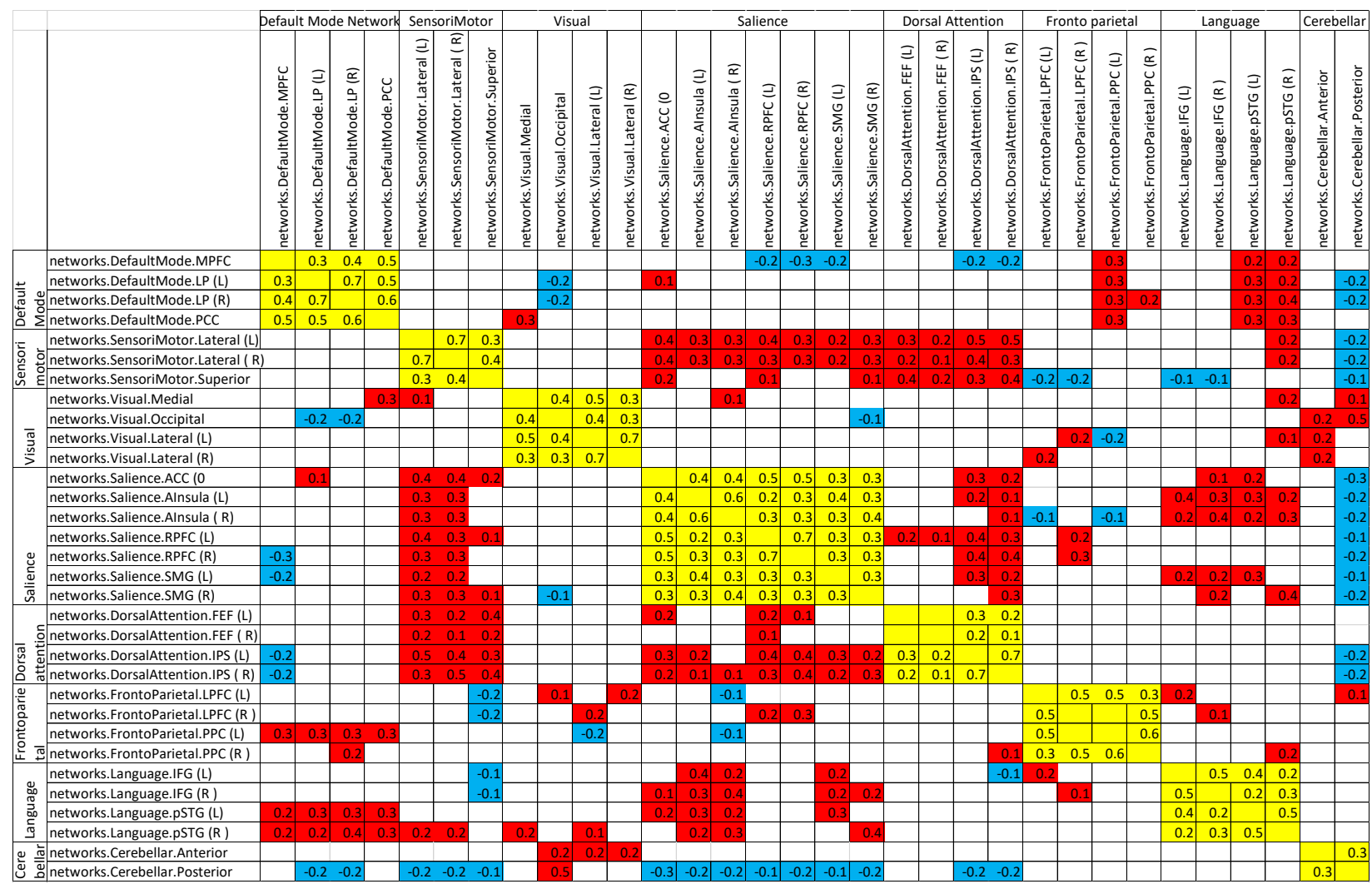

Fig. 2: Connectivity matrix for main effects of STP for condition shapepresent 
Table I: Comparison of main effects of LTP and STP for various condition contrasts

\begin{tabular}{|c|c|c|c|c|c|c|}
\hline \multirow[b]{2}{*}{ Condition contrast } & \multicolumn{3}{|c|}{ Simple main effect of LTP } & \multicolumn{3}{|c|}{ Simple main effect of STP } \\
\hline & Seed ROI & Target ROI & beta & Seed ROI & Target ROI & beta \\
\hline \multirow[t]{4}{*}{ Rare $>$ Freq } & $\begin{array}{l}\text { Visual } \\
\text { Medial } \\
\end{array}$ & Insula (L) & -0.12 & \multirow{4}{*}{\multicolumn{3}{|c|}{ No significant connectivity }} \\
\hline & PPC(L) & RPFC (R ) & 0.1 & & & \\
\hline & PPC(L) & pSTG (R) & -0.008 & & & \\
\hline & $\mathrm{LPFC}(\mathrm{R})$ & pSTG(L) & -0.10 & & & \\
\hline \multirow[t]{3}{*}{ Cdoff $>$ Cdon } & SMG(R ) & $\mathrm{FEF}(\mathrm{R})$ & -0.14 & $\mathrm{LP}(\mathrm{R})$ & $\begin{array}{l}\text { Visual lateral } \\
\text { (R ) }\end{array}$ & -0.18 \\
\hline & IPS(L) & $\mathrm{PPC}(\mathrm{L})$ & -0.15 & $\mathrm{LP}(\mathrm{R})$ & $\begin{array}{l}\text { Cerebellar } \\
\text { posterior }\end{array}$ & 0.16 \\
\hline & $\begin{array}{l}\text { Cerebellar } \\
\text { posterior }\end{array}$ & PPC(L ) & 0.14 & PCC & $\begin{array}{l}\text { Visual lateral } \\
\text { (R) }\end{array}$ & -0.14 \\
\hline $\begin{array}{l}\text { Face absent }>\text { face } \\
\text { present }\end{array}$ & DMN.LP(L) & DMN.LP(R ) & 0.2 & \multicolumn{3}{|c|}{ No significant connectivity } \\
\hline $\begin{array}{l}\text { Shape absent > } \\
\text { shape present }\end{array}$ & \multicolumn{3}{|c|}{ No significant connectivity } & Insula (L) & IFG(R ) & -0.21 \\
\hline \multirow[t]{2}{*}{ Active $>$ baseline } & DMN.LP(R ) & LPFC(L) & 0.14 & DMN.LP(L) & $\begin{array}{l}\text { Visual lateral } \\
\text { (R) }\end{array}$ & -0.28 \\
\hline & $\begin{array}{l}\text { Visual lateral } \\
\text { (L) }\end{array}$ & $\begin{array}{l}\text { Visual lateral } \\
\text { (R) }\end{array}$ & -0.24 & $\begin{array}{l}\text { Visual lateral } \\
\text { (L) }\end{array}$ & $\mathrm{PPC}(\mathrm{L})$ & -0.23 \\
\hline
\end{tabular}

Fig. 4: Connectivity pattern of STP group

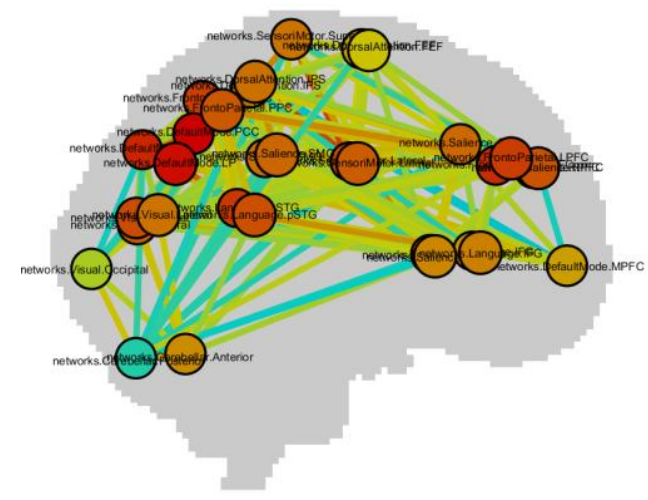

Fig. 3: Connectivity pattern of LTP group

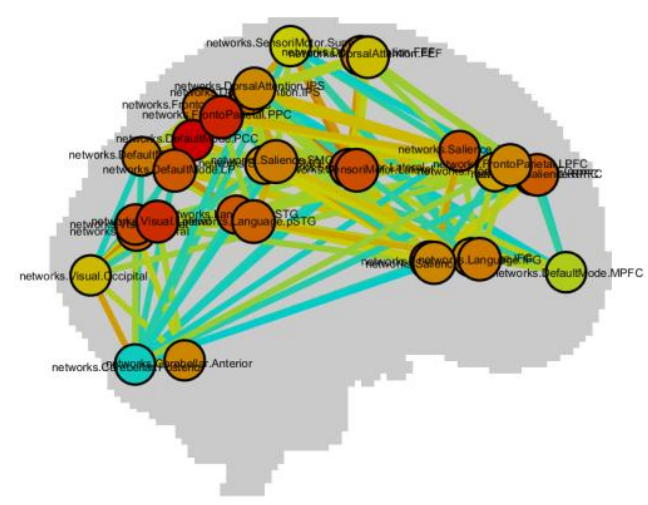

\section{CONCLUSION}

A benefit of meditative practice is that it appears to be effective in improving one's attention. Are the effects of meditation altered by the number of years of meditation practice also needs to be explored. In this study, a comparison is made between long term meditation practitioners (LTP) and short-term meditation practitioners (STP) to see the differences and similarities in the connectivity patterns. From the analysis, it is evident that in fact there are certain differences in connectivity between long term and short term practitioners. Hence continuous practice of meditation can have long term effects.

\section{ACKNOWLEDGMENT}

We gratefully thank Visvesvaraya Technological University, Jnana Sangama, Belagavi for the support extended to this research work. We also like to extend our gratitude to BNM Institute of Technology for the continuous support and encouragement. 


\section{REFERENCES}

1. Bryan Williams, "A Glimpse into the Meditating Brain", Morning Star Center for Spiritual Living, June 7, 2009.

2. NairA. K., Sasidharan A., John J. P., Mehrotra S., Kutty B. M., "Assessing Neurocognition via Gamified Experimental Logic: A Novel Approach to Simultaneous Acquisition of Multiple ERPs", Frontiers in Neuroscience, 2016, 10

3. E. Baron Short, Samet Kose, Qiwen Mu, Jeffery Borckardt, Andrew Newberg, Mark S. George, and F. Andrew Kozel, "Regional Brain Activation during Meditation Shows Time and Practice Effects: An Exploratory FMRI Study", Advance Access Publication 27 December 2007

4. J.A. Brefczynski-Lewis, A. Lutz, H.S. Schaefer, D.B. Levinson, R.J. Davidson, Neural correlates of attentional expertise in long-term meditation practitioners, Proc. Natl. Acad. Sci. U.S.A. 104 (2007) 11483-11488

5. B.K. Hölzel, U. Ott, H. Hempel, A. Hackl, K. Wolf, R. Stark, D Vaitl, Differential engagement of anterior cingulate and adjacent medial frontal cortex in adept meditators and non-meditators, Neurosci. Lett. 421 (2007) 16-21.

6. Joon Hwan Jang,Wi Hoon Jung, Do-Hyung Kang, Min Soo Byun,Soo Jin Kwon, Chi-Hoon Choi, Jun Soo Kwon, "Increased default mode network connectivity associated with meditation", Neuroscience Letters 487 (2011) 358-362

7. Whitfield-Gabrieli S., Nieto-Castanon A. "Conn: A functional connectivity toolbox for correlated and anticorrelated brain networks", Brain Connectivity, 2012, doi:10.1089/brain.2012.0073

\section{AUTHORS PROFILE}

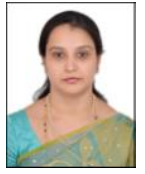

Ashwini S Savanth received post graduate degree from Visveswaraya Technological University, Belgaum in 2006. She is currently working as an Assistant Professor in the Dept. of E \& C, BNMIT, Bangalore and is pursuing research. Her areas of interest are Image Processing, Pattern Recognition and Digital Signal Processing.

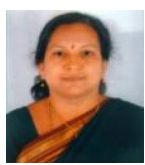

Dr.P.A.Vijaya received Ph.D from IISc, Bangalore in 2005 She is currently working as a Professor \& HoD in Dept. of E $\&$ C, BNMIT, Bangalore. She has around 90 publications in reputed journals and conferences. She is guiding 6 research students. Her areas of interest are Pattern Recognition, Image Processing and embedded systems.

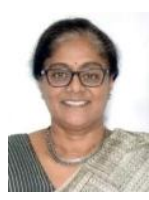

Dr.Bindu M. Kutty received Ph.D in Neurophysiology She is currently working as a Professor in the department of Neurophysiology. Her areas of specialization include Neurobiology of meditation, cognition and consciousness, Neurobiology of sleep and wake, Neurobiology of Schizophrenia, Neurobiology of learning and memory. 